\title{
Hydrogen in Tungsten as Plasma-facing Material
}

\author{
Joachim Roth, Klaus Schmid
}

Max-Planck-Institut für Plasmaphysik, EURATOM-Association, 85748 Garching, Germany

\begin{abstract}
:
Materials facing plasmas in fusion experiments and future reactors are loaded by high fluxes $\left(10^{20}\right.$ to $\left.10^{24} \mathrm{~m}^{-2} \mathrm{~s}^{-1}\right)$ of $\mathrm{H}, \mathrm{D}$, T fuel particles at energies between few $\mathrm{eV}$ to $\mathrm{keV}$. In this respect, the evolution of the radioactive $\mathrm{T}$ inventory in the first wall, the permeation of $\mathrm{T}$ through the armour into the coolant and the thermo-mechanical stability after long term exposure are key parameters determining the applicability of a first wall material.

W exhibits fast hydrogen diffusion, but an extremely low solubility limit. Due to the fast diffusion of hydrogen and the short ion range most of the incident ions will quickly reach a surface and recycle into the plasma chamber. For steady state operation the solute hydrogen for typical fusion reactor geometry and wall conditions can reach an inventory of about $1 \mathrm{~kg}$. However, in short pulse operation typical for ITER, solute hydrogen will diffuse out after each pulse and the remaining inventory will consist of hydrogen trapped in lattice defects, such as dislocations, grain boundaries, and irradiation induced traps. In high flux areas the hydrogen energies are too low to create displacement damage. However, under these conditions the solubility limit will be exceeded within the ion range and the formation of gas bubbles and stress induced damage occurs. In addition, simultaneous $\mathrm{n}$ fluxes from the nuclear fusion reaction $\mathrm{D}(\mathrm{T}, \mathrm{n}) \alpha$ will lead to damage in the materials and produce trapping sites for diffusing hydrogen atoms throughout the bulk. The formation and diffusive filling of these different traps will determine the evolution of the retained $\mathrm{T}$ inventory.

The presentation will concentrate on experimental evidence for the influence of different trapping sites on the hydrogen inventory in $\mathrm{W}$ as studied in ion beam experiments and low temperature plasmas. Based on the extensive experimental data base models are applied to estimate the contribution of different trap to the influence of different traps in future fusion reactors. 1 page
\end{abstract}




\section{Introduction}

The first wall in fusion devices is exposed to a high flux $\left(10^{20}\right.$ to $\left.10^{24} \mathrm{~m}^{-2} \mathrm{~s}^{-1}\right)$ of hydrogen isotopes $(\mathrm{H}, \mathrm{D}, \mathrm{T})$ at energies ranging from $\mathrm{eV}$ to $\mathrm{keV}$. Apart from challenges arising due to erosion induced life time limitations and the general thermo-mechanical loading conditions, the retention of hydrogen species in the fist wall is of key concern. Retention of large amounts of $\mathrm{T}$ would result in large radioactive inventory after shut down of the device which poses a safety hazard. Also $\mathrm{T}$ is a precious resource which is bred from $\mathrm{Li}$ in the fusion device blanket and a high retention of $\mathrm{T}$ in the first wall would degrade the fuelling efficiency of the burning plasma.

Therefore ideally the first wall material should retain as little hydrogen as possible. A possible candidate material is $\mathrm{W}$ which features a very low solubility for hydrogen among other favourable properties like low physical sputtering yield and high melting temperature. However the actual amount of hydrogen that can be retained in $\mathrm{W}$ is determined by the defect density in the material. These defects act as trap sites for hydrogen where it is retained after exposure to the plasma while excess, solute hydrogen diffuses out the material even at room temperature. The number of trap sites depends strongly on the grade i.e. the structure of the W material. Typical natural trap site densities in the $\mathrm{W}$ bulk are in the order of $10^{-4}$ and lower. This natural defect density can be increased by radiation damage both by the incident hydrogen ions and by the fast $\mathrm{n}$ from the $\mathrm{D}(\mathrm{T}, \mathrm{n}) \alpha$ fusion reaction. While the damage by ions is limited to the near surface regions, the fast $\mathrm{n}$ produces trap sites throughout the $\mathrm{W}$ bulk which can greatly enhance the potential for hydrogen retention. The ions not only produce damage as part of the collision cascade at ion energies above the displacement threshold but also at low energies where the over saturation of the implantation zone leads to high stress fields which produces cracks and gas filled cavities. These defects both degrade the thermo mechanical stability and act as trap sites for hydrogen.

Whether all the natural and generated traps are filled and thus become a problem, strongly depends on the operating conditions of the fusion device in particular on the temperature of the first wall. The build up of the hydrogen inventory by filling the traps is driven by diffusion of hydrogen from the implantation zone ( ion range) into the $\mathrm{W}$ bulk. The release of hydrogen from $\mathrm{W}$ is determined by thermally activated de-trapping of hydrogen from the trap sites and subsequent diffusion towards the surface where it is released by recombining with another hydrogen atom. For fusion relevant conditions, i.e. high fluxes and temperatures, this release is not limited by the recombination process but by the diffusive transport of hydrogen towards the surface. Therefore, with increasing temperature both the release of hydrogen and 
the filling of traps in the bulk are increased and a prediction about the retained amount of hydrogen requires detailed modelling of the underlying diffusion trapping and trap generation processes. Despite the fact that the validation of these models is still ongoing and not all experimental observations are fully understood, they are the only means to extrapolate from the laboratory experiments to the different operating conditions in future fusion devices like ITER and ultimately DEMO.

This paper will focus on the influence of different defects sites on the retention of hydrogen in W. After reviewing the transport parameters of hydrogen in $\mathrm{W}$, the contribution of intrinsic defects to the hydrogen inventory is discussed. Then the production of defects is reviewed focusing on the structural modifications by the high flux ion bombardment, the influence of simultaneous $\alpha$ irradiation on the damage structure, and the cascade damage by the high energy $n$ from the $D(T, n) \alpha$ fusion reaction. Finally modelling results are presented that allow an extrapolation of laboratory experiments to the different operating conditions in ITER and later DEMO.

\section{Transport parameters for $\mathrm{H}$ in $\mathrm{W}$}

$\mathrm{W}$ is an endothermal material for the uptake of H. Fig. 1 shows the schematic potential diagram for $\mathrm{H}$ in $\mathrm{W}$. Atomic hydrogen can penetrate the surface potential, while molecular hydrogen must be dissociatively adsorbed before penetrating the lattice. In the lattice diffusion is thermally activated. Hydrogen reaching the surface and being adsorbed at the surface can be released as molecules after recombining with another adsorbed atom. In the bulk, $\mathrm{H}$ can be trapped at trapping sites, e.g. grain boundaries, dislocations, vacancies, or gas bubbles in the lattice. For modelling the transport of hydrogen in tungsten reliable data for the diffusion coefficient, the recombination rate coefficient, the solubility as well as trap densities and activation energies are needed.

The status of the data for these parameters was reviewed recently by Causey et al $\left[{ }^{1}\right]$, summarising experimental values for the diffusion coefficient, recombination rate coefficient and solubility. The most commonly accepted expression for the diffusivity is the one reported by Frauenfelder $\left[{ }^{2}\right]$ in 1969 (see table 1). Frauenfelder's experiments were performed on samples with the lowest intrinsic damage level and at temperatures where trapping would have no effect on the hydrogen migration. Other investigators $\left[{ }^{3}, 4\right]$ did acknowledge that including trapping effects in their analysis might result in both data sets agreeing with the earlier extrapolated results of Frauenfelder [2]. This may also be the case for more recent data 
from Gasparyan et al. $\left[{ }^{5}\right]$ measured in permeation experiments. Data obtained by modelling reemission and thermal desorption of deuterium from VPS tungsten coatings $\left[{ }^{6}{ }^{7}\right]$ used multiple parameters to fit the release data thus yielding values for the diffusivity no more accurate than the values assumed for the other parameters entering the evaluation (e.g. trapping and recombination rate coefficients). For the diffusivity of hydrogen in tungsten, the expression reported by Frauenfelder [2] should be used.

\begin{tabular}{|l|l|l|l|l|}
\hline $\begin{array}{l}\text { Recommended values } \\
{[12]}\end{array}$ & $\begin{array}{l}\text { Activation } \\
\text { energy } \\
{[\mathrm{eV}]}\end{array}$ & $\begin{array}{l}\text { Pre-exponential } \\
\text { factor }\end{array}$ & $\begin{array}{l}\text { Trap } \\
\text { concentration } \\
{[\text { at\%] }}\end{array}$ & Reference \\
\hline Diffusion coefficient & -0.39 & $4.1 \times 10^{-7}\left[\mathrm{~m}^{2} / \mathrm{s}\right]$ & & Frauenfelder [2] \\
\hline $\begin{array}{l}\text { Recombination rate } \\
\text { coefficient }\end{array}$ & 1.16 & $3.2 \times 10^{-15}\left[\mathrm{~m}^{4} / \mathrm{s}\right]$ & & Anderl $\left[^{8}\right]$ \\
\cline { 2 - 5 } & -2.06 & $3 \times 10^{-25} / \mathrm{T}^{1 / 2}\left[\mathrm{~m}^{4} / \mathrm{s}\right]$ & & Pick et al. $\left[{ }^{9}\right]$ \\
\hline Solubility & -1.04 & $9 \times 10^{-3}\left[\mathrm{H} / \mathrm{W} \mathrm{atm}^{1 / 2}\right]$ & & Frauenfelder [2] \\
\hline Intrinsic traps & 0.85 & & $4 \times 10^{-4}$ to $10^{-2}$ & $\begin{array}{l}\text { Ogorodnikova } \\
{\left[{ }^{10}\right]}\end{array}$ \\
\hline Ion induced traps & 1.45 & & up to 10 & $\begin{array}{l}\text { Ogorodnikova } \\
{[8]}\end{array}$ \\
\hline n-induced traps & $1.8-2.2$ & & up to 1 & Tyburska $\left[{ }^{11}\right]$ \\
\hline
\end{tabular}

The rate of surface recombination of hydrogen from tungsten is more difficult to quantify (Fig. 2). There are two reports of very low values [6,7]. As stated above for the diffusion coefficient, the coefficients derived by these researchers were based on multi-parameter fittings of retention and thermal release results. On the other hand, there are relatively high values reported by Anderl et al. [8] determined by fitting permeation data. In such a study examining steady state permeation, the number of parameters affecting the results is less than that for the above studies where retention and thermal desorption were analyzed. Tritium retention data over the temperature range of $423-973 \mathrm{~K}$ by Causey et al. $\left[{ }^{12}\right]$, as well as data by Haasz et al. $\left[{ }^{13}\right]$ showed low retention and rapid release indicating that the release was not rate limited by recombination, implying a $\mathrm{C}=0$ boundary condition. Recent data from Gasparyan et al $\left[{ }^{14}\right]$ from ion-driven permeation through $\mathrm{W}$-foils confirm the range the magnitude of the data by Anderl, but cover too small a temperature range to determine the temperature dependence. Values calculated using the Pick and Sonnenberg model [9] are much larger than all experimental values. Their model was derived under the assumption of a clean surface which means that chemisorption activation energy, $\mathrm{E}_{\mathrm{C}}$, was set equal to zero. Choosing different values for $\mathrm{E}_{\mathrm{C}}$, e.g. due to adsorbed surface impurities, strongly influences the recombination rate and can invert the temperature dependence for $E_{C}>1 \mathrm{eV}$. However, overall magnitude is not so important [1] as in fusion conditions with incident fluxes 
$>10^{22} / \mathrm{m}^{2} \mathrm{~s}$ any recombination rate coefficient greater than about $10^{-24} \mathrm{~m}^{4} / \mathrm{s}$ represents almost instantaneous recombination at the surface. Calculations to predict hydrogen isotope retention in tungsten will not significantly underestimate the retention if the boundary condition $\mathrm{C}=0$ (large $\mathrm{K}_{\mathrm{R}}$ ) is applied at all boundaries.

The database for reported solubility in tungsten is very limited $\left[2,{ }^{15}\right]$. As with his diffusivity measurements, Frauenfelder [2] saturated $99.95 \%$ pure tungsten samples with hydrogen over the temperature range 1100-2400 K. Mazayev et al. [15] examined the solubility of hydrogen in tungsten over a more limited temperature range (1900 to about $2400 \mathrm{~K})$. At these higher temperatures, his measured solubility agrees very well with the measurements of Frauenfelder $[2]$.

\section{Schematics of hydrogen retention in $\mathrm{W}$}

Assuming clean W surfaces under intense hydrogen fluxes, i.e. diffusion limited surface conditions, the steady state solute $\mathrm{H}$ concentration in the $\mathrm{W}$ will establish linear gradients from the depth of implantation to the front and back surface with very low surface concentrations. In steady state the incident flux (minus reflected flux) must balance the outflux from all surfaces. Due to the short ion range compared with the $\mathrm{W}$ thickness most of the emitted flux will originate at the front surface. The maximum lattice concentration $\mathrm{c}_{\max }$ of solute hydrogen at the depth of the ion range $\mathrm{R}_{\mathrm{p}}$ will establish such that the gradient towards the front surface establishes a flux to the surface roughly equal to the incident ion flux $\mathrm{I}_{\mathrm{o}}$, resulting in

$$
\mathrm{c}_{\max }=\left(\mathrm{I}_{\mathrm{o}} \cdot \mathrm{R}_{\mathrm{p}}\right) / \mathrm{D}
$$

It must be noted that values for $\mathrm{c}_{\max }$ obtained in using typical fusion relevant values exceed by far the thermodynamics solubility and are equivalent to internal gas pressures of many GPa. The surface concentration, while being low, must allow for the recombination of hydrogen atoms to molecules for reemission. The permeation flux equals in first approximation the incident flux times the ratio of ion range to foil thickness, typically of the order of $10^{-4}$. The solute inventory and the accompanying permeating fluxes were intensely discussed and assessed in the 1980's with review papers by Doyle et al. $\left[{ }^{16}\right]$ and Möller et al. $\left[{ }^{17}\right]$, taking also conditions of recombination limitation into account, as well as diffusion in the presence of temperature gradients for a large variety of fusion relevant metals. In later publications, the effect of trapping of hydrogen in lattice damage was sketchily taken into account, adding little to the steady state inventory in the walls. 
In a steady state fusion device, the main inventory may indeed be the total amount of solute tritium retained in the $\mathrm{W}$ walls. For ITER, with pulse lengths of about $400 \mathrm{~s}$, the solute inventory will be released and recovered between discharges, while the long term inventory is just the fraction stably retained in traps at the given operation temperatures. Therefore, trap sites in the lattice must be thoroughly discussed, i.e. intrinsic traps in the material (grain boundaries, dislocations), the formation of ion induced traps (Frenkel pairs at high enough ion energies, stress induced dislocation networks, gas bubbles) and eventually n-induced traps (atom displacements (dpa), damage clusters).

\section{Traps formation for hydrogen in $\mathrm{W}$}

\section{Intrinsic defects}

Under intrinsic defects we understand hydrogen trapping sites in previously un-irradiated material. Intrinsic defects can be grain boundaries, pores and inclusions, dislocations or thermal vacancies. Clearly, the intrinsic defect concentration can vary drastically depending on material grade and purity, on thermal pre-treatment and surface preparation. The microstructure of tungsten samples was systematically modified by recrystallization to investigate the structure dependence of deuterium (D) retention. $\left[{ }^{18}\right]$. The modification by recrystallization allowed a wide variation in the crystallite size and had a strong impact on the measured D retention. Both the total amount and the binding state of the retained D were changed. Generally, W coating material, such as VPS layers, contain much larger amounts of retained hydrogen $\left[{ }^{19}\right]$. Relatively high D concentration (above 0.1 at.\%) at depths of several micrometers were observed after D plasma exposure at 340-560 K and could be related to accumulation of $\mathrm{D}_{2}$ molecules in pores, while at temperatures above $600 \mathrm{~K}$ deuterium is accumulated mainly in the form of $\mathrm{D}$ atoms chemisorbed on the inner pore surfaces. Similarly, very high levels of trap concentrations of the order of $10^{-2}$ at $\%$ were observed in porous W/Re-alloys $\left[{ }^{20}\right]$.

The effect of grain boundaries can best be investigated in a comparison of polycrystalline $\mathrm{W}$ with single crystalline material. Haasz et al $\left[{ }^{21},{ }^{22}\right]$ and Alimov et al $\left[{ }^{23}\right]$ have recently investigated the hydrogen retention in $\mathrm{W}$ single crystals and polycrystalline material. In both investigations the incident $\mathrm{D}$ ion energy was well below the threshold for displacement damage which is of the order of $1 \mathrm{kev}$. Best information on the effect of intrinsic damage can be obtained from depth profiles of $\mathrm{D}$, distinguishing near surface trapping sites 
from intrinsic bulk damage. Fig. 3 shows a comparison of poly- and single crystalline $\mathrm{W}$ from ref [23].

The depth at which deuterium is retained can be tentatively divided into three zones: (i) the near-surface layer (up to a depth of $\sim 0.2 \mu \mathrm{m}$ ), (ii) the sub-surface layer (from $\sim 0.5$ to $\sim 2 \mu \mathrm{m}$ ), and (iii) the bulk $(>5 \mu \mathrm{m})$. At temperatures above $400 \mathrm{~K}$ D diffuses into the bulk and decorates intrinsic traps. At this depth the $\mathrm{D}$ concentration at high ion fluences $\geq 1 \cdot 10^{24} \mathrm{D} / \mathrm{m}^{2}$ varies from below $10^{-4}$ at $\%$ for $\mathrm{W}$ single crystal to $3 \cdot 10^{-3}$ at $\%$ for polycrystalline $\mathrm{W}$. This difference must be attributed to grain boundaries and can account for a plasma-facing material thickness of $10 \mathrm{~mm}$ for most of the integral retention of about $10^{22} / \mathrm{m}^{2}$ observed at high fluences above $10^{24} / \mathrm{m}^{2}$. On the other hand, in the surface and sub-surface zones very similar trap densities develop during hydrogen irradiation in single crystals as in polycrystalline material even at a sub-threshold ion energy of $38 \mathrm{eV}$, and the formation mechanism will be discussed below.

\section{Hydrogen induced defects}

As shown in Fig. 3 the implantation of low energy D ions into W material creates trapping sites in the sub-surface layer extending many $\mu \mathrm{m}$ into the material, i.e. far beyond the actual range of the ions. One often discussed possibility results from the high pressures equivalent to the large lattice concentrations of solute hydrogen within the implantation range in relation to the low solubility of hydrogen in W. Plastic deformation of the W matrix caused by deuterium super-saturation within the near-surface layer is assumed to result in bubble and void formation. A review of hydrogen bubbles in metals by Condon and Schober $\left[{ }^{24}\right]$ outlines some possible cavity formation mechanisms. During irradiation the D concentration in the implantation zone greatly exceeds the solubility limit and stresses the matrix lattice until plastic deformation with formation of voids and vacancy clusters occurs to alleviate these tensions.

In sputter-depth profiling after high flux D implantation combined with residual gas analysis molecular $\mathrm{D}_{2}$ was found to be released from surface near layers, leading to the conclusion of the formation of hydrogen gas bubbles $\left[{ }^{25}\right]$. However, direct observation of bubbles due to hydrogen implantation was not possible at low ion fluxes and fluences in ion beam experiments, even in high resolution TEM. Typically a dense network of dislocation resulted from the irradiation $\left[{ }^{26}\right]$. Only post-irradiation annealing resulted in the formation of gas bubbles $\left[{ }^{27}\right]$. After $8 \mathrm{keV} \mathrm{D}$ irradiation and annealing to temperatures above $873 \mathrm{~K} \mathrm{D}$ agglomerated into nano-size gas bubbles. 
Recently, at much higher fluxes and fluences surface and sub-surface structures could be resolved. Recrystallized polycrystalline tungsten was exposed to a deuterium plasma with high flux of $10^{22} \mathrm{D} / \mathrm{m}^{2} \mathrm{~s}$ of $\approx 38 \mathrm{eV}$ up to fluences of $10^{26}$ and $10^{27} \mathrm{D} / \mathrm{m}^{2}$ and temperature between 320 and $800 \mathrm{~K}$. The observed surface topography, showing blister-like structures $\left[{ }^{28},{ }^{29},{ }^{30}\right]$, varies with temperature, fluence and depends strongly on the grain orientation. By cross-sectioning with a FIB through the blister-like structures on the surface, their subsurface morphology and position is accessible and analysed using SEM.

With increasing temperature, the observable cracks below the surface increase in length, number and width. At $\approx 360 \mathrm{~K}$ the fine crack-like defects inside grains show a length $\approx 5 \mu \mathrm{m}$ and width $\approx 0.1 \mu \mathrm{m}$ up to a depth of $\approx 10 \mu \mathrm{m}$. Around the cracks often large distorted areas are observed (Fig. 4a). At higher temperatures (500-650 K), large cavities at the grain boundaries together with their large surface structures are the dominating features and the fine-crack structures with the distorted region around lose their significance (Fig. 4b). For temperatures $>700 \mathrm{~K}$, no blister-like structures are detected. In most cases a correlation of shape and position of the defect structures underneath a surface structure with the crystallite orientation is obvious, especially at temperatures between 500 and $650 \mathrm{~K}$ for the large cavities at the grain boundaries. An explanation could be that tungsten behaves as brittle at temperatures less than $\approx 500 \mathrm{~K}$ and the stress by the high transient $\mathrm{D}$ inventory during implantation deforms plastically the grains in the distorted area and creates cracks within the grains. At higher temperatures (up to $650 \mathrm{~K}$ ) the brittle to ductile transition $\left[{ }^{31},{ }^{32}\right]$, which is dependent on stress and on material parameters like the dissolved hydrogen content, reduces cracking, and large cavities arise only on the grain boundaries $\left[{ }^{33}\right]$. Material can be moved easily from cavities to surface bulges along gliding planes corresponding to the low-index slip system $\{110\}<111>$ in order to reduce mechanical stress by transient D inventory. This means gliding along the $<111>$ direction in the $\{110\}$ planes. The movement may be supported by Das pressure in the cavity from the super-saturated bulk inventory. Therefore the surface structures are elongated along the gliding plane and the position of resulting surface features relative to the cavity is determined by the slip system direction. This process forms surface structures which reflect the shape of the cavity $\left[{ }^{34}\right]$.

The observation of cracks, distorted areas, and large cavities beneath the surface of Dimplanted W indicates that the stress during D implantation exceeds the toughness of the material due to a $\mathrm{D}$ concentration exceeding the solubility $\left[{ }^{35}, 21,{ }^{36},{ }^{37}\right]$. The weak points in the material could be the defects, like vacancies, voids, dislocations and grain boundaries, on 
which the deuterium can agglomerate $\left[{ }^{38},{ }^{39}\right]$. The coincidence of the maximum in the temperature dependence of the D retention $\left[{ }^{40}\right]$ (Fig. 5) with the appearance of the highest density of surface topographical changes is evident (see Fig 6a).

The absence of blister-like structures as well as the low D retention at temperatures above $700 \mathrm{~K}$ indicates that the requirement for extended defect production is not fulfilled. Possibly, it could be explained by the strongly increased solubility and diffusivity $\left[{ }^{41}\right]$ avoiding the transient D concentration exceeding the solubility. Also it could be speculated that the defects in $\mathrm{W}$ anneal at these temperatures, the re-emission is increased, and therefore, the transient D concentration is strongly lowered, in consequence reducing stress and strain for defect production.

\section{Influence of $\alpha$ irradiation on hydrogen retention}

Due to fusion reactions in the central plasma $\alpha$-particles are created. After thermalisiation in the plasma He ions will impinge onto the plasma facing materials together with hydrogen ions and a He concentration of 5 to $10 \%$ can be assumed in the incident flux.

Earlier experiments on deuterium implantation after He pre-irradiation of metals at energies of several keV showed an enhancement of the retention of hydrogen isotopes $\left[{ }^{42}, 43\right]$. T A trapping mechanisms related to He bubbles were suggested. However, the He bubbles have been shown to act both as trapping sites for $\mathrm{D}$ and as diffusion barriers for $\mathrm{D}$ depending on the relative depth of implantation of $\mathrm{D}$ and He, respectively $\left[{ }^{44},\right]$. In the case of simultaneous irradiation of PCW by $500 \mathrm{eV}$ helium and deuterium ions the presence of He enhanced D trapping in the near surface, while limiting D diffusion into the bulk $\left[{ }^{45}\right]$.

In the case of low-energy helium-seeded deuterium plasma exposure, the deuterium retention in polycrystalline $\mathrm{W}$ was reduced compared to only pure deuterium plasma $\left[26,{ }^{46}\right]$. At high fluxes and fluences above $10^{26} \mathrm{D} / \mathrm{m}^{2}$ seeding of helium ions into the $\mathrm{D}$ plasma significantly reduces the $\mathrm{D}$ retention at elevated temperatures (400-700K) (Fig. 5) and prevents the formation of blisters (Fig. 6). The structure below the surface is strongly modified by the additional He irradiation. According to Iwakiri et al $\left[{ }^{47}\right]$, in W irradiated with low-energy He ions, He platelets and bubbles are formed in the implantation zone. Based on results obtained for simultaneous irradiation of $\mathrm{W}$ with helium and hydrogen ions, Ueda et al $\left[{ }^{48}\right]$ have concluded that hydrogen atoms can be trapped at the periphery of $\mathrm{He}$ bubbles thus reducing 
the hydrogen inward flux into the bulk. He bubbles formed in the near-surface layer under exposure to the He-seeded D plasmas could increase the stress field around the high-pressure bubbles generating defects in the crystal lattice $\left[{ }^{49},{ }^{50}\right]$. In doing so, the defects could serve as trapping sites for D atoms such that deuterium is accumulated only in the near-surface layer [45].. On the other hand, a dynamic mechanism of nano-scale helium bubble formation can lead to development of open porosity in the near-surface layer and create pathways to the surface thus enhancing the D re-emission thus limiting the D diffusion into the bulk. Additionally, the porous near-surface structure may serve as a damper layer to dissipate the compressive stresses induced by the local deuterium super-saturation. As consequence, no D trapping sites are generated in the sub-surface layer.

\section{Neutron induced defects}

The prediction of hydrogen isotope retention in n-irradiated tungsten (W) is an important topic for edge plasma research as it has significant implications for the operation of ITER and fusion reactors. Numerical simulation of tritium retention in n-induced irradiation defects has been the subject of recent review papers $\left[{ }^{51}, 52\right]$ pointing out the need for further experimental investigations. However, direct measurement of tritium retention in n-irradiated tungsten is a difficult task. The self-implantation of $\mathrm{W}$ is the most promising approach because it generates dense cascades with large clusters which are typical for n-irradiation and any chemical effects are avoided. Pre-damaged samples were exposed to deuterium plasmas on both front (undamaged) and rear (damaged) sides in order to separate deuterium (D) retention in plasmainduced traps and in displacement damage created by fast tungsten ions $\left[{ }^{53}\right]$. Irradiation was done up to a damage level expected from the end-of-life neutron fluence in ITER.

The results show that $\mathrm{D}$ retention in self-damaged tungsten correlates with a damage level calculated by SRIM program. Thermal desorption spectroscopy reveals a new trapping site, stronger than sites in not pre-irradiated material, indication trapping in vacancy clusters and voids. The trapping energies of $\mathrm{D}$ with radiation-induced vacancies and vacancy clusters were found to be $1.45 \mathrm{eV}$ and $1.85 \mathrm{eV}$, respectively $\left[{ }^{54}, 52\right]$. It has been found that radiation traps are saturated with dpa (Fig. 7, $\left[54,{ }^{55},{ }^{56}, 57\right]$ ) where the saturated number of traps produced by n fluence $(\approx 0.7 \mathrm{dpa})$ depends on temperature. The saturated trap density saturates at about $1.2 \%$ at $470 \mathrm{~K}$ and decreases to less than 1 at. $\%$ for $\mathrm{T}>500 \mathrm{~K}$. The rate at which such traps are filled during plasma exposure was derived from permeation experiments when the plasma exposure was done on undamaged (front) side and the decoration of radiation damage was 
measured on the damaged (rear) side at different fluences [53]. The effect of deuterium trapping by strong and weak defects was explored through plasma exposures at different temperatures. Trapping of $\mathrm{D}$ in radiation damage is reduced with temperature because weak traps cannot hold any D and damage sites are annealed.

In order to describe deuterium migration in $\mathrm{W}$, a diffusion model with trapping in different kinds of defects was used. The deuterium depth profiles and the release kinetic during heating were successfully described for undamaged and pre-damaged W. The diffusion model with dynamic trap formation during irradiation was validated by comparison with experiments and applied for calculation of hydrogen isotope retention in ITER in the presence of neutron irradiation.

\section{Consequences for ITER and DEMO:}

Already in ITER, but especially in DEMO the fuel retention properties of W plasma-facing materials will be enhanced due to radiation damage after high fluence n-irradiation, which provides additional trapping sites for hydrogen. The irradiation damage at the end of the ITER lifetime has been estimated to $0.6 \mathrm{dpa}$ in the divertor and $1 \mathrm{dpa}$ at the first wall $\left[{ }^{58}\right]$, but the microstructure and its relation to hydrogen trapping is largely unknown. Simulations using the DIFFUSE code $\left[{ }^{59}\right]$ build-up of n-induced trapping sites to a saturation value of $0.6 \%$ deduced very similar retention values as simulations in ref. $\left[{ }^{60}\right]$ assuming saturation at $1 \%$ (Fig. 7 ). In these calculations no ion induced trap generation has been taken into account leading to a retention increase with the square root of fluence. Consequently, a value of $700 \mathrm{~g}$ retained tritium will be reached after about 5000-10000 discharges [ $\left.{ }^{61}\right]$ (Fig. 8, [52,51]). Here the diffusion limited solution for hydrogen release has been taken from ref. [51]. The data for not pre-damaged $\mathrm{W}$ follow the retention curve and the associated uncertainties as evaluated in ref $\left[{ }^{62}\right]$.

The saturation concentration of n-produced trap of $1 \%$ in $\mathrm{W}$ is an extreme upper limit and probably $0.1 \%$ is a more realistic value for ITER walls at elevated temperatures. Taking into account a saturation of damage sites at $0.01 \%$ after $0.6 \mathrm{dpa}$ as reported for Mo $\left[{ }^{63}\right]$, the additional trapping sites due to neutron damage might not be limiting throughout the lifetime of ITER. In addition, the density of n-induced vacancies will decrease with temperature by increasing the spontaneous annihilation and vacancy clustering. As such effects are not taken into account the present estimations give upper limits of $\mathrm{T}$ retention and need to be refined. In 
spite of the coarse inclusion of the n-damage effect in the present modelling, both assessments show remarkable agreement. In both cases the unirradiated $\mathrm{W}$ retention is very similar, being dominated by the divertor areas at moderate fluxes and temperatures. Clearly, the additional effect due to $n$-damage requires more experimental validation and more detailed code simulation before a final conclusion can be drawn $\left[{ }^{64}\right]$.

While the tritium inventory for ITER may not constitute a major problem, long term operation in DEMO will lead to permeation of hydrogen isotopes through the W surface coating on the coolant structure. A further parameter to be taken into account in future experimental studies are thermal and stress gradients in a thin $(\mathrm{mm}) \mathrm{W}$ armour on the coolant structure and associated interfacial problems.

However, as dominant consequence of the high fluence irradiation with hydrogen isotopes, the structural stability of a W armour may be severely jeopardised. Depending on operational temperature, but especially at typical ITER wall temperatures of about $500 \mathrm{~K}$, brittle intragranular cracks of large cavities resulting from dislocation gliding to the surface will appear which can act as nuclei for further crack growth under the expected thermal transients due to ELMs or disruptions. While for the case of ITER experimental irradiation data are available covering fluences expected within the lifetime of ITER PFMCs, for the steady state operation in DEMO much larger fluences are expected and no experimental basis exists. The additional high $\mathrm{n}$ fluences will also change the bulk properties of $\mathrm{W}$ materials and make predictions for DEMO materials very uncertain. Calculations show that the $\mathrm{T}$ inventory does not reach the limit of $700 \mathrm{~g}$ within the lifetime of $\mathrm{W}$ components in ITER for the all-W assumption. However, there remain considerable uncertainties concerning D retention in tungsten, such as the estimate of the divertor and wall fluxes, effect of transient heat loads and the effect of simultaneously implanted He.

\section{Acknowledgement}

The authors want to acknowledge the collaboration, support and many stimulating discussions with all colleagues in the plasma-wall interaction group at IPP Garching. We thank in particular Vladimir Alimov, Martin Balden, Karl Ertl, Stephan Lindig, Armin Manhard, Olga Ogorodnikova and Beata Tyburska. 



\section{Figure Captions:}

Fig. 1: Schematic potential energy diagram for $\mathrm{H}$ in $\mathrm{W}$. The indicated energy terms are explained in the text.

Fig. 2: Recombination rate coefficient for hydrogen in tungsten.

Fig. 3: Depth profile of $200 \mathrm{eV}$ D implanted into single- and poly-crystalline $\mathrm{W}$ to affluence of $10^{24} / \mathrm{m}^{2}$ at different temperatures [24]

Fig 4: Surface and sub-surface structures in $\mathrm{W}$ after irradiation with $10^{27} \mathrm{D} / \mathrm{m}^{2}$ at $38 \mathrm{eV}$ and a flux of $10^{22} \mathrm{D} / \mathrm{m}^{2} \mathrm{~s}$ at temperatures below ( a ) and above ( $\mathrm{b}$ ) the ductile to brittle transition temperature [34] The arrow in ( $b$ ) indicates the direction of material transport from the cavity to surface structure.

Fig. 5: Temperature dependence of $\mathrm{D}$ retention in $\mathrm{W}$ after exposure to pure $\mathrm{D}$ plasmas and $\mathrm{D} / 5 \%$ He plasmas. The difference of NRA to TDS results indicates the amount of D retained in the bulk at depths larger then $7 \mu \mathrm{m}[41]$.

Fig. 6: SEM images of re-crystallized W exposed to pure D plasma (38 eV/D) (a) and heliumseeded D plasma (38eV/D + $76 \mathrm{eV} / \mathrm{He}, 5 \%$ of He ions) [41].

Fig. 8: Tritium retention in ITER assessed for all-W PFCs without (shaded red area) and with n-damage (lines) to the wall material. 
Fig. 1: Schematic potential energy diagram for $\mathrm{H}$ in $\mathrm{W}$. The indicated energy terms are explained in the text.

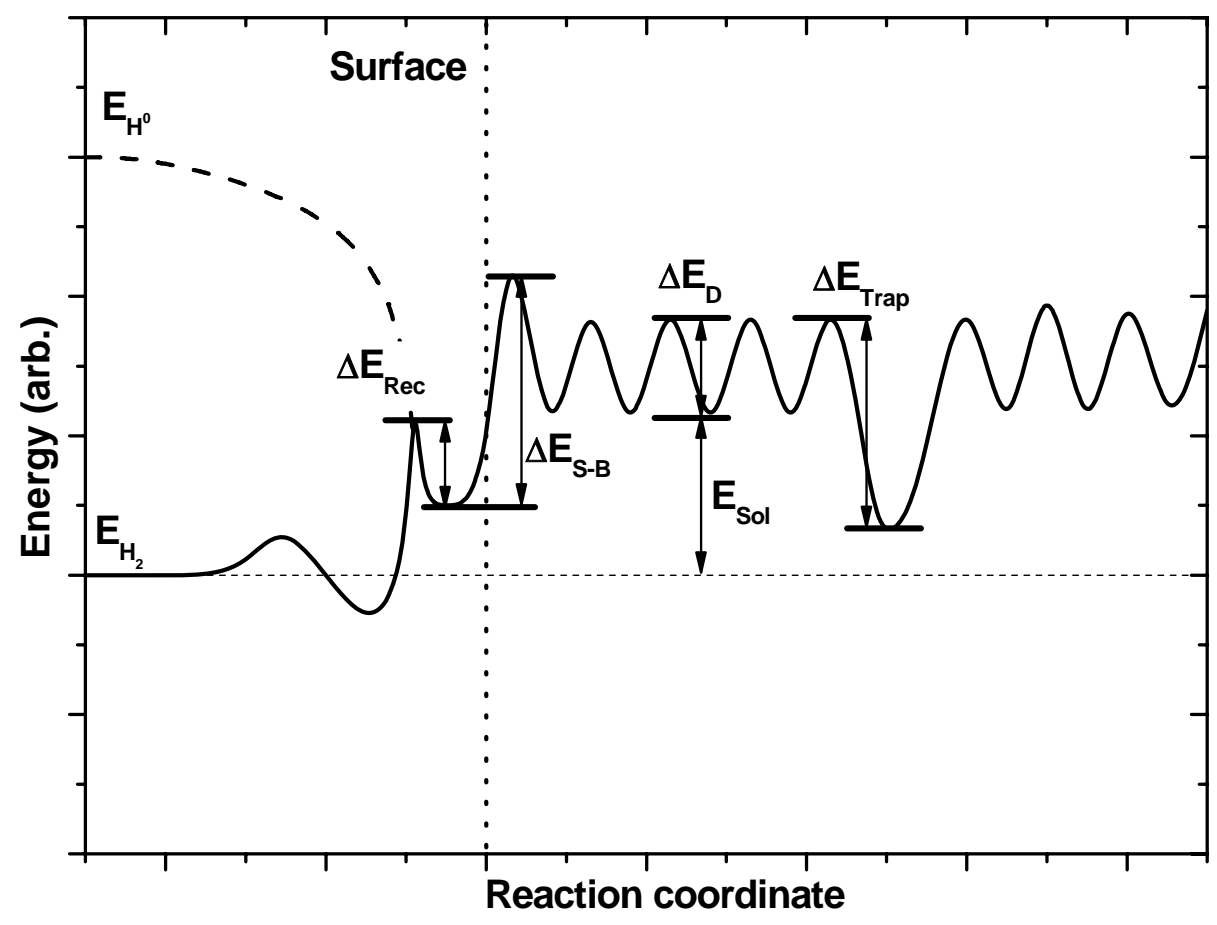


Fig. 2: Recombination rate coefficient for hydrogen in tungsten.

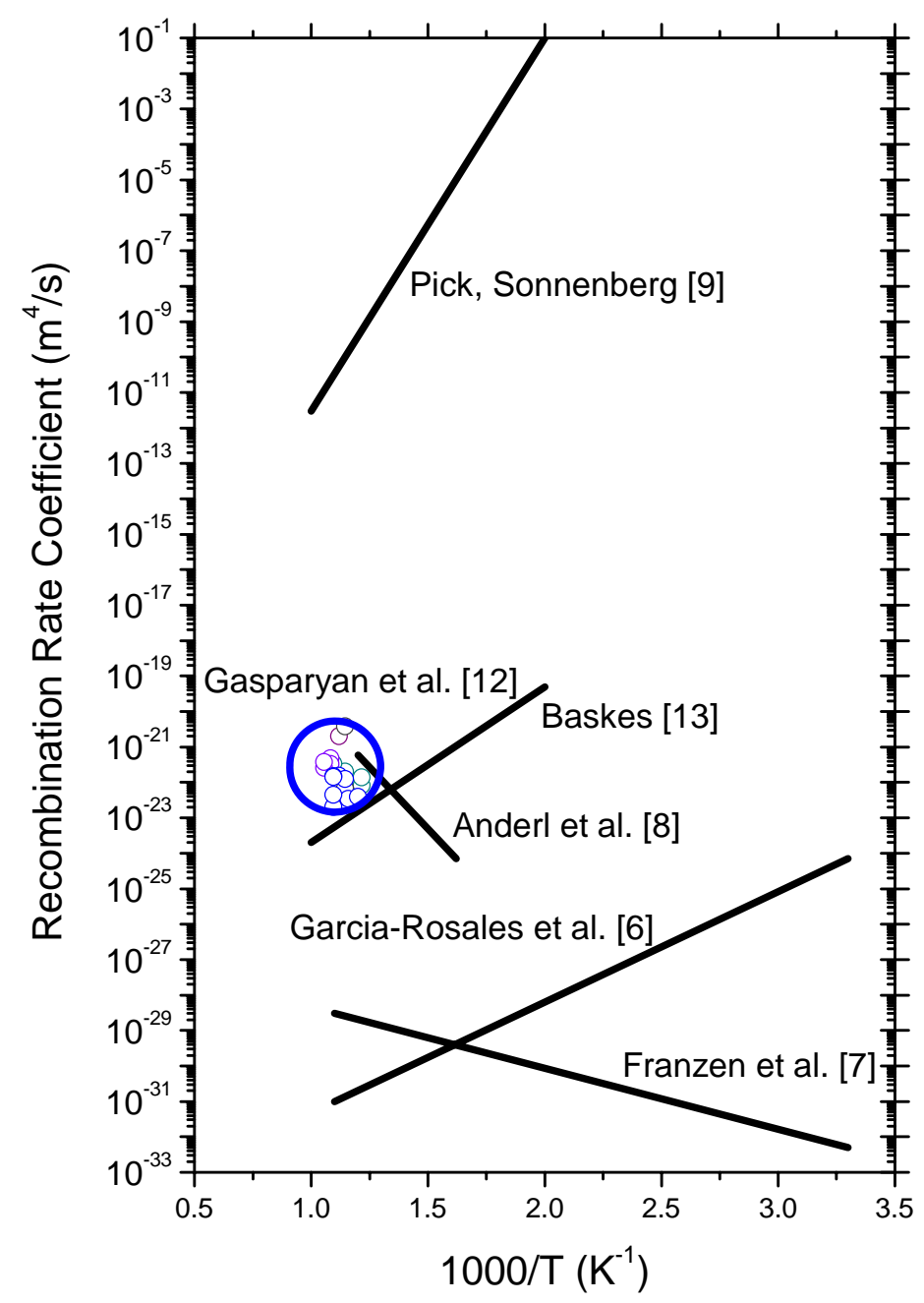


Fig. 3: Depth profile of $200 \mathrm{eV}$ D implanted into single- and poly-crystalline $\mathrm{W}$ to affluence of $10^{24} / \mathrm{m}^{2}$ at different temperatures [24].
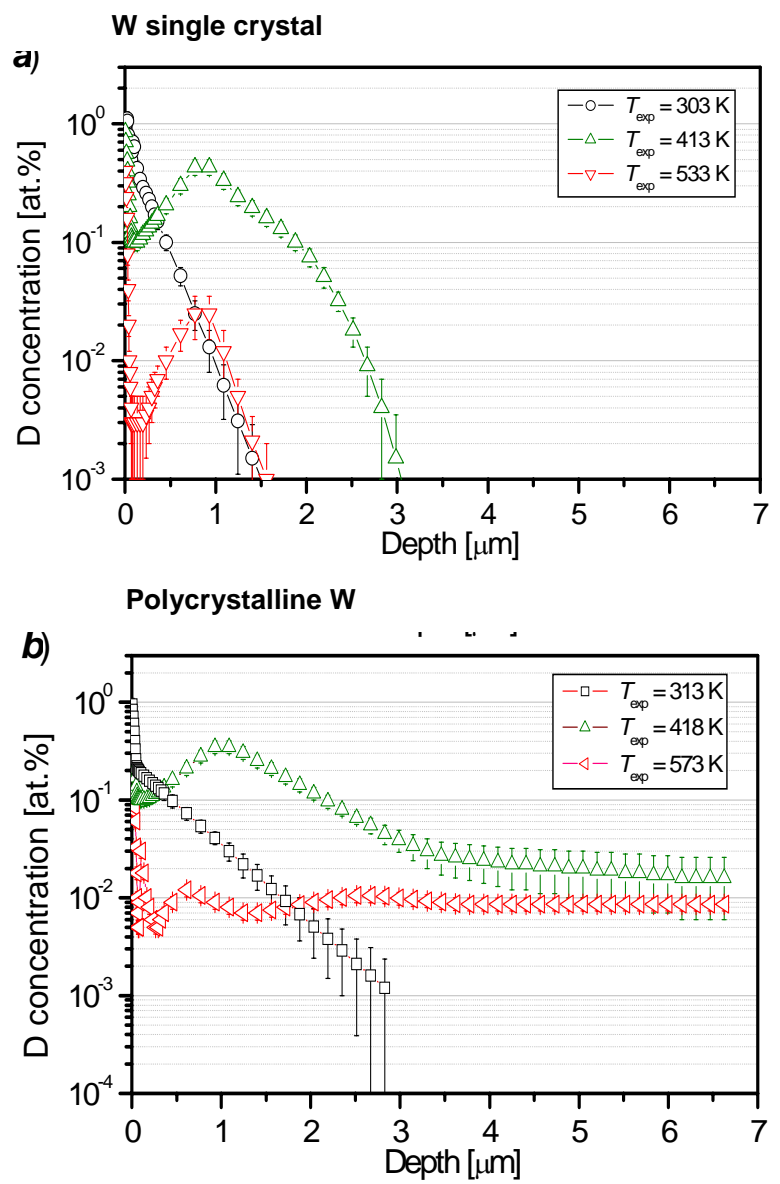
Fig 4: Surface and sub-surface structures in $\mathrm{W}$ after irradiation with $10^{27} \mathrm{D} / \mathrm{m}^{2}$ at $38 \mathrm{eV}$ and a flux of $10^{22} \mathrm{D} / \mathrm{m}^{2} \mathrm{~s}$ at temperatures below ( a ) and above ( b ) the ductile to brittle transition temperature [34] The arrow in ( b ) indicates the direction of material transport from the cavity to surface structure.
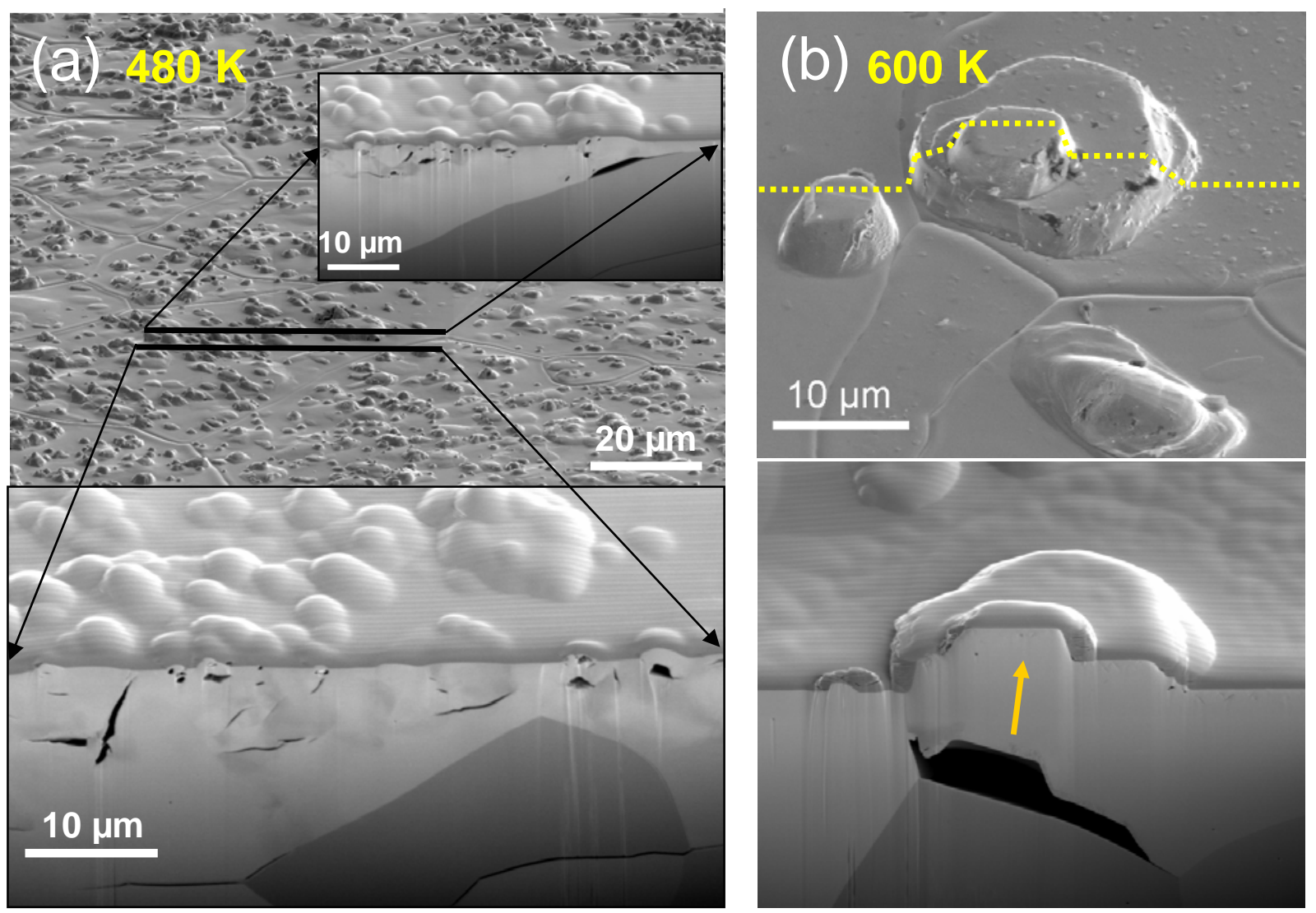
Fig. 5: Temperature dependence of $\mathrm{D}$ retention in $\mathrm{W}$ after exposure to pure $\mathrm{D}$ plasmas and $\mathrm{D} / 5 \%$ He plasmas. The difference of NRA to TDS results indicates the amount of D retained in the bulk at depths larger then $7 \mu \mathrm{m}$. [41]

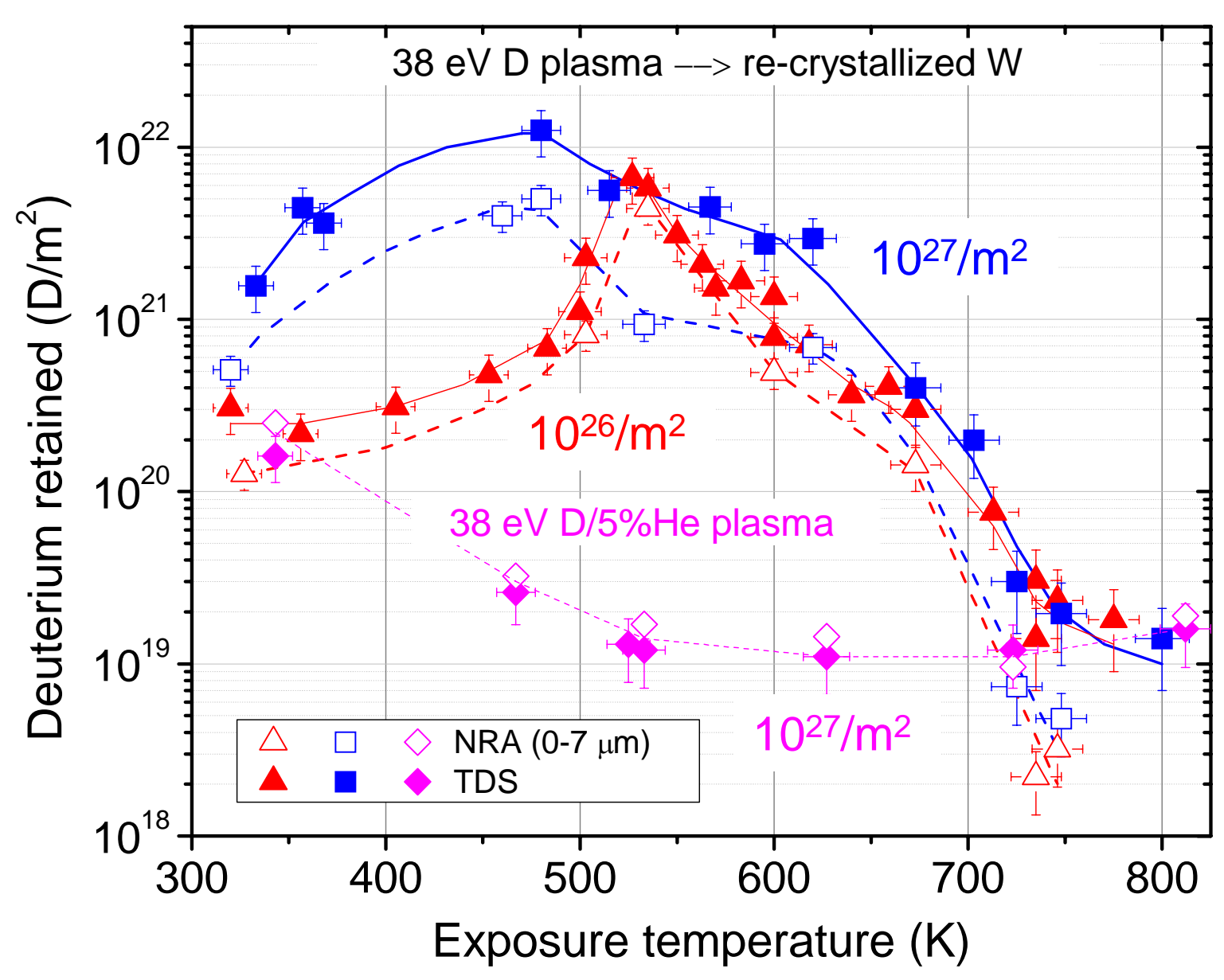


Fig. 6: SEM images of re-crystallized W exposed to pure D plasma (38 eV/D) (a) and heliumseeded D plasma (38eV/D + $76 \mathrm{eV} / \mathrm{He}, 5 \%$ of He ions) [41].
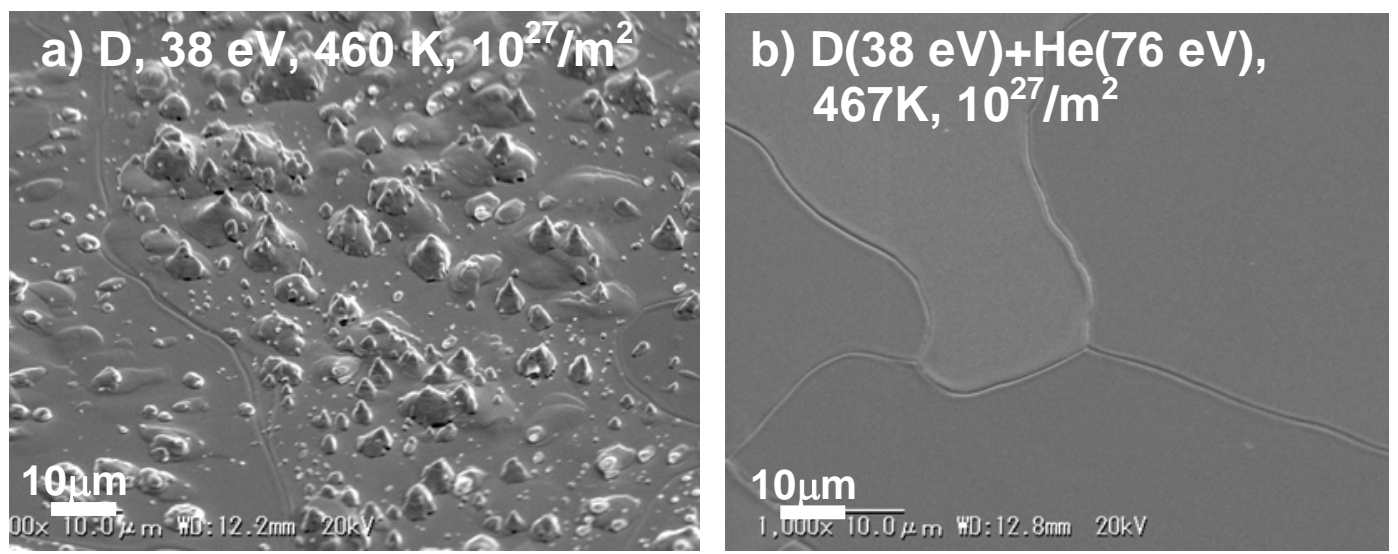
Fig. 7: Dependence of hydrogen trap density in $\mathrm{W}$ as function of dpa induced by irradiation with different high energy heavy ions [56].

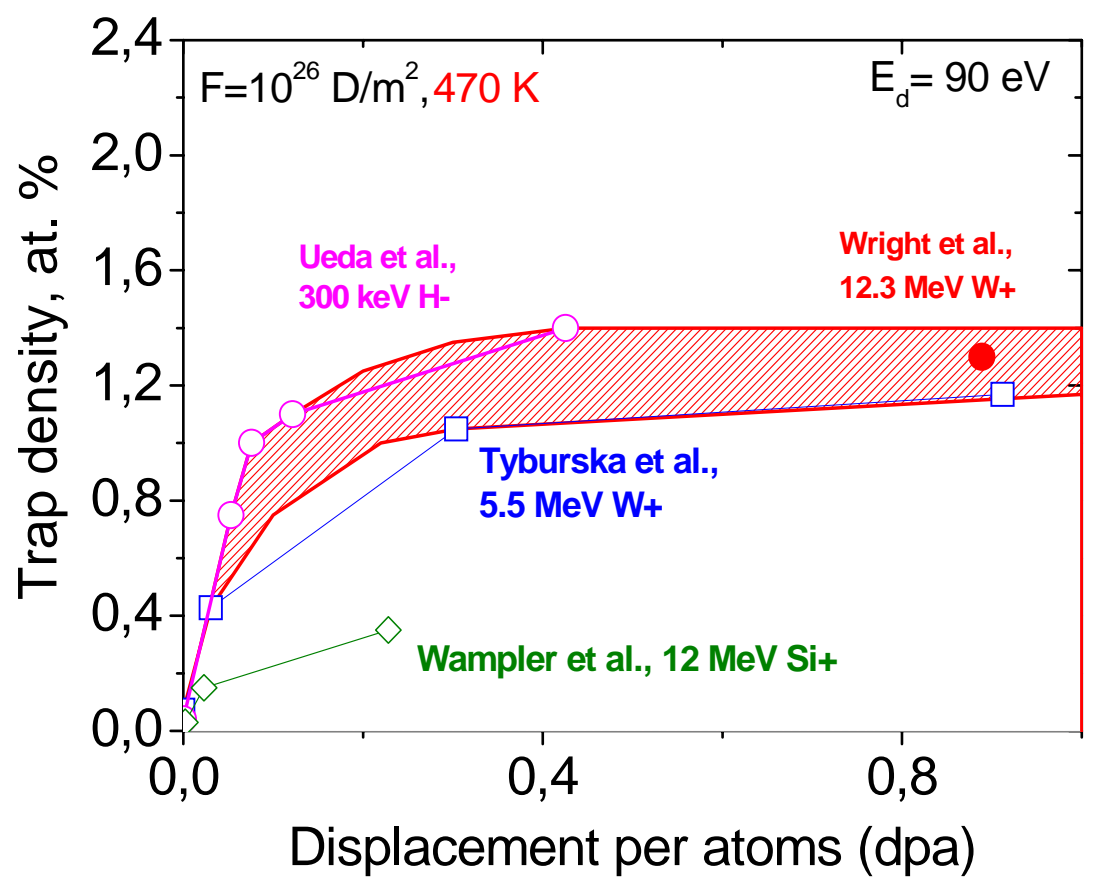


Fig. 8: Tritium retention in ITER assessed for all-W PFCs without (shaded red area) and with n-damage (lines) to the wall material.

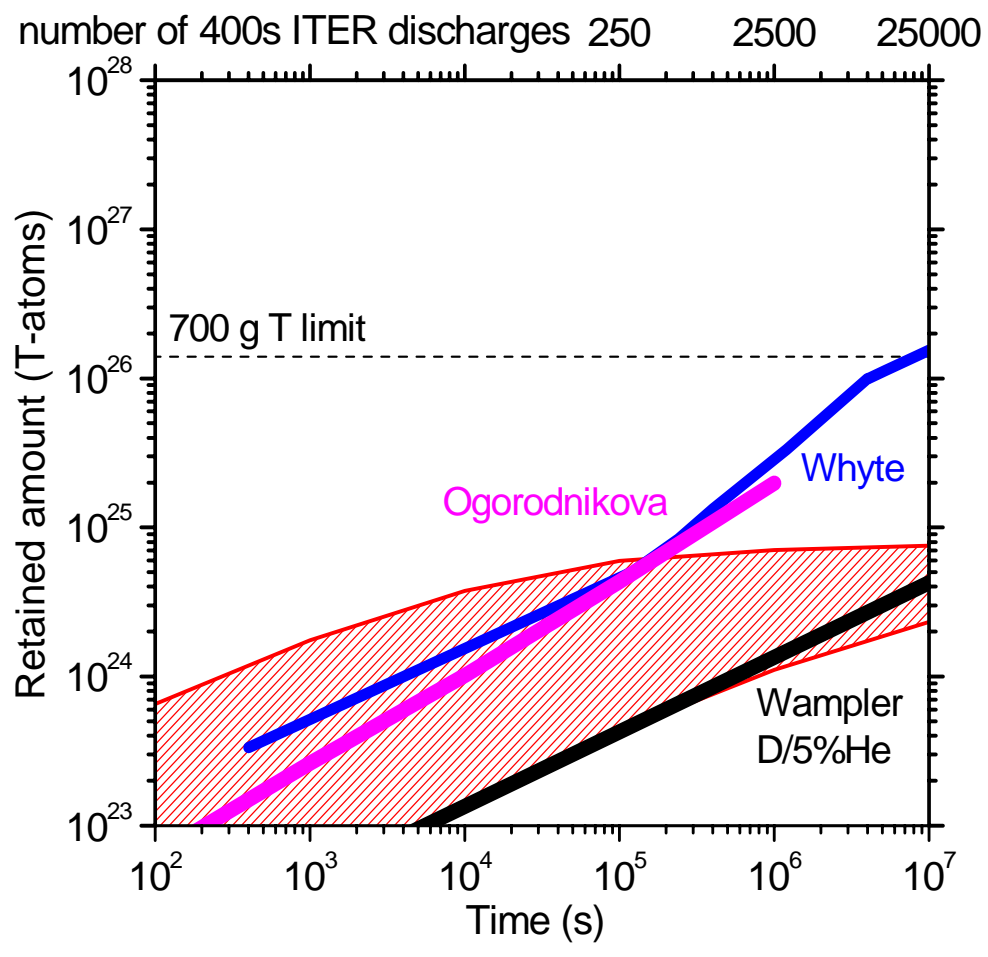




\section{References:}

${ }^{1}$ Rion A. Causey and Thomas J. Venhaus, Physica Scripta T94 (2001) 9

${ }^{2}$ R. Frauenfelder, J. Vac. Sci. Technol. 6 (3) (1969) 388.

${ }^{3}$ G. Benamati, E. Serra, C.H. Wu, J. Nucl. Mater. 283-287 (2000) 1033

${ }^{4}$ A.P. Zakharov, V.M. Sharapov, E.I. Evko, Fiz. Khim. Mekh. Mater. 9 (2) (1973) 29

${ }^{5}$ Yu.M. Gasparyan, A.V. Golubeva, M. Mayer, A.A. Pisarev, J. Roth, J. Nucl. Mater. 390-391 (2009) 606

${ }^{6}$ C. Garcia-Rosales, P. Franzen, H. Plank, et al., J. Nucl. Mater. 233-237 (1996) 803

${ }^{7}$ P. Franzen, C. Garcia-Rosales, H. Plank, V.Kh. Alimov, J. Nucl. Mater. 241-243 (1997) 1082.

${ }^{8}$ R.A. Anderl et al., Fus. Technol. 21 (1992) 745

${ }^{9}$ M.A. Pick, K. Sonnenberg, J. Nucl. Mater. 131 (1985) 208

${ }^{10}$ O.V. Ogorodnikova, J. Roth, M. Mayer, J. Nucl. Mater. 313-316 (2003) 469.

${ }^{11}$ B. Tyburska, V.Kh. Alimov, O.V. Ogorodnikova, K. Schmid, K. Ertl, J. Nucl. Mater 395 (2009) 150.

${ }^{12}$ R. Causey et al., J. Nucl. Mater. 266-269 (1999) 467

${ }^{13}$ A.A. Haasz et al., J. Nucl. Mater. 258-263 (1998) 889

${ }^{14}$ Yu.M. Gasparyan, A.V. Golubeva, M. Mayer, A.A. Pisarev, J. Roth, J. Nucl. Mater. 390-391 (2009) 606

${ }^{15}$ A. Mazayev et al., Izv. Akad. Nauk. USSR Metally 6 (1968) 233

${ }^{16}$ B. L. Doyle and D. K. Brice, J. Nucl. Mater. 122 \& 123 (1984) 1523-1530

${ }^{17}$ W. Möller and J. Roth, in: Physics of Plasma-Wall Interactions in Controlled Fusion. Plenum Press (1986) p. 439

${ }^{18}$ A. Manhard, K. Schmid, M. Balden, W. Jacob, Influence of the microstructure on the deuterium retention in tungsten, JNM (2011) in press

${ }^{19}$ V.Kh. Alimov, H. Nakamura, B. Tyburska-Püschel, O.V. Ogorodnikova, J. Roth, K. Isobe, T. Yamanishi, J. Nucl. Mater., In Press, Available online 27 May 2011

${ }^{20}$ A.V. Golubeva, et al., JNM 363-365 (2007) 893

${ }^{21}$ A.A. Haasz, M. Poon, J.W. Davis, J. Nucl. Mater. 266-269 (1999) 520

${ }^{22}$ M. Poon et al., J.Nucl. Mater. 337-339 (2005) 629

${ }^{23}$ V.Kh. Alimov J. Roth, M. Mayer, J. Nucl. Mater. 337-339 (2005) 619-623

${ }^{24}$ J.B. Condon, T. Schober, J. Nucl. Mater. 207 (1993) 1

${ }^{25}$ V.Kh. Alimov, Phys.Scr. T108 (2004) 46

${ }^{26}$ M. Miyamoto et al., Nucl. Fusion 49 (2009) 065035

${ }^{27}$ R. Sakamoto, T. Mutoga, N. Yoshida, J. Nucl. Mater. 220-222 (2005) 819

${ }^{28}$ W. Wang, J. Roth, S. Lindig, C.H. Wu, JNM 299(2001)124

${ }^{29}$ M. Balden, S. Lindig, A. Manhard, V.Kh, Alimov, O.V. Ogorodnikova, J. Roth, this conference P51A

${ }^{30}$ S. Lindig, M. Balden, V.Kh. Alimov, A. Manhard, C. Höschen, T. Höschen, this conference P63B

${ }^{31}$ P. Gumbsch et al., Science $282(1998) 1293$

${ }^{32}$ A. Giannattasio et al., Phys. Scr. T128 (2007) 87

${ }^{33}$ S. Lindig et al, Phys. Scr. T138 (2009) 014040

${ }^{34}$ S Lindig, M Balden, V Kh Alimov, T Yamanishi, WM Shu and J Roth, Phys. Scr. T138 (2009) 014040

${ }^{35}$ C.H Skinner et al., Fusion Sci. Technol. 54 (2008) 891

${ }^{36}$ W.M. Shu, Appl. Phys. Lett. 92 (2008) 211904

${ }^{37}$ V.Kh. Alimov et al., J. Nucl. Mater. 375 (2008) 192

${ }^{38}$ H. Eleveld and A. van Veen, J. Nucl. Mater. 191-194 (1992) 433

${ }^{39}$ A. van Veen, et al., J. Nucl. Mater. 155-157 (1988) 1113

${ }^{40}$ V.Kh. Alimov et al., Surface morphology and deuterium retention in tungsten exposed to low-energy, high-flux pure and helium-seeded deuterium plasmas Phys. Scr. T138 (2009) 014048

${ }^{41}$ R.A. Causey and T.J. Venhaus, Phys. Scr. T94 (2001) 9

${ }^{42}$ S. Nagata, B. Tsuchiya, T. Sugawara, N. Ohtsu, and T. Shikama, Nucl. Instrum. Methods Phys. Res. B 190 (2002)652

${ }^{43}$ H. T. Lee, A. A. Haasz, J. W. Davis, and R. G. Macaulay-Newcombe, J. Nucl. Mater. 360 (2007) 196

${ }^{44}$ H. T. Lee, A. A. Haasz, J. W. Davis, R. G. Macaulay-Newcombe, D. G. Whyte, and G. M. Wright, J. Nucl. Mater. 363-365 (2007) 898

${ }^{45}$ V. Kh. Alimov, W. M. Shu, J. Roth, K. Sugiyama, S. Lindig, M. Balden, K. Isobe, and T. Yamanishi, Phys. Scr. T138 (2009) 014048

${ }^{46}$ Y. Ueda, H. Kashiwagi, M. Fukumoto and N. Yoshida, Fusion Sci. Technol. 56 (2009) 85

${ }^{47}$ H. Iwakiri et al., J. Nucl. Mater. 283-287 (2000) 1134

${ }^{48}$ Y. Ueda Y et al., J. Nucl. Mater. 386-388 (2009) 725

${ }^{49}$ H. Iwakiri, K. Morishita and N. Yoshida J. Nucl. Mater. 307-311 (2002) 135

${ }^{50}$ H.T. Lee et al., J. Nucl. Mater. 363-365 (2007) 898

${ }^{51}$ D.G. Whyte, J. Nucl. Mater. 390-391 (2009) 911.

${ }^{52}$ O. Ogorodnikova et al., J. Nucl. Mater. (2011) in press, PSI San Diego

${ }^{53}$ B. Tyburska, V. Kh. Alimov, O.V. Ogorodnikova, K. Ertl, K. Schmid, J. Roth, J. Nucl. Mater. (2011) in press, 
${ }^{54}$ B. Tyburska, V.Kh. Alimov, O.V. Ogorodnikova, K. Schmid, K. Ertl, J. Nucl. Mater. 395 (2009) 150

${ }^{55}$ W.R.. Wampler, R. Doerner, Nucl. Fusion 49 (2009) 115023

${ }^{56}$ G.M. Wright, M. Mayer, K. Ertl, G. de Saint-Aubin, J. Rapp, TMAP7 simulations of deuterium trapping in preirradiated tungsten exposed to high-flux J. Nucl. Mater., In Press, Available online 7 January 2011

${ }^{57}$ M. Fukumoto, H. Kashiwagi, Y Ohtsuka, Y.Ueda et al. J. Nucl. Mater. 390-391 (2009) 572

${ }^{58}$ Materials Assessment Report G 74 MA 10 01-07-11 W, Ch. 2.2 Tungsten, ITER, 2001

${ }^{59}$ M.I. Baskes, Sandia Nat. Lab. Livermore, Report SAND80-8201, 1980

${ }^{60}$ O. Ogorodnikova, ITPA SOL/DIV Garching, 200

${ }^{61}$ J. Roth et al., PPFC 50 (2008) 103001

${ }^{62}$ B. Lipschultz, J. Roth, et al., MIT Rep. PSFC/RR-10-4 (2010)

${ }^{63}$ M. Eldrup, M. Li, L. Snead, S. Zinkle, Nucl. Instr. Methods in Phys. Research, B266 (2008) 3602

${ }^{64}$ J. Roth et al., J. Nucl. Mater. 390-391 (2009) 1-9 